\title{
Performance Analysis of Existing Routing Protocols
}

\author{
R. Kumari ${ }^{*}$, P. Nand ${ }^{2}$, \\ ${ }^{1 *}$ Dept. of Computer Science and Engineering, Galgotias University, Gr. Noida, India \\ ${ }^{2}$ Dept. of Computer Science and Engineering, Galgotias University, Gr. Noida, India \\ "Corresponding Author: ranichoudhary04@gmail.com, Tel.: 9971733493
}

Available online at: www.isroset.org

Received $14^{\text {th }}$ Sep 2017, Revised 17 $7^{\text {th }}$ Sep 2017Accepted 24 $4^{\text {th }}$ Oct 2017, Online $30^{\text {th }}$ Oct 2017

\begin{abstract}
Keeping in mind the end goal to break down and look at the execution of the four steering conventions AODV, DSR, DYMO and ZRP, reproduction tests are performed. The motivation behind the re-enactments is to test the proficiency of the steering conventions under various system conditions and system sizes. The emphasis is focused on five execution measurements: Packet conveyance proportion, normal Jitter, end-to-end deferral, throughput and vitality utilization.
\end{abstract}

Keywords-CBR;DYMO;AODV; ZRP;

\section{INTRODUCTION}

Parcel conveyance proportion: It is the proportion of the quantity of information bundles effectively conveyed to the goal to the aggregate number of information parcels sent by source hubs.

Normal Jitter: As the parcels from source to goal will achieve the goal with various deferrals, the bundle's postponement changes with its position in the lines of the switches along the way amongst source and goal and this position can shift erratically. This variety in delay is known as Jitter. Jitter can truly influence the nature of gushing sound and additionally video. A system could have zero Jitter. Jitter for all the priority bits are figured and looked at.

End-to-End delay: End-to-end defer demonstrates the period of time taken for a parcel to go from the CBR (Constant Bit Rate) source to the goal. It speaks to the normal information postpone an application or a client encounters when transmitting information.

Throughput: Throughput is the normal rate of fruitful message conveyance over the correspondence channel. It is measured in bits every second (piece/s or bps) and some of the time in information parcels every second or information bundles per schedule vacancy. Because of changing heap of different clients having a similar system assets, the bit-rate (the greatest throughput) that can be furnished with a specific information stream might be too low for constant media administrations if all information streams get a similar planning need.

Vitality Consumption: This is the measure of vitality devoured by Motes amid the times of transmit, get, sit still and rest states. The unit of vitality utilization utilized as a part of the recreations is $\mathrm{mJ}$.

\section{RESULTS AND ANALYSIS}

The reproduction parameters are recorded in Table 1 . The CBR movement is accepted with the accompanying normal parcel rates: 0.1 bundle for every second (pps), $0.2 \mathrm{pps}, 1$ pps, 5 pps and 10 pps are utilized. The reproduction comes about have been arrived at the midpoint of more than 10 distinctive seed esteems from 1 to 10 .

\begin{tabular}{|c|c|}
\multicolumn{2}{|c}{ Table 1. Simulation Parameters } \\
\hline Parameters & Values \\
\hline Nodes & 33 \\
\hline Terrain Area & $100 \mathrm{~m} * 100 \mathrm{~m}$ \\
\hline Transmission range & 35 meter \\
\hline TX-Power & $0 \mathrm{dBm}$ \\
\hline Path Loss Model & Two Ray Model \\
\hline PHY and MAC Model & IEEE 802.15 .4 \\
\hline Energy Model & MICAZ Mote \\
\hline Battery Model & Simple Linear,1200 mAhr \\
\hline Payload size & 50 bytes \\
\hline
\end{tabular}

In Star topology, DSR has high parcel conveyance proportion and AODV has a poor Packet conveyance proportion. The correlation of steering conventions in star topology is given in Table 1. DYMO and ZRP has parcel conveyance proportion in the middle of them. The Average Packet conveyance proportion in Star topology is around 
$60 \%$. Normal Jitter esteem is high for low movement load and declines as the activity stack increments. This is a direct result of the expansion in the parcel era rate. ZRP has low normal jitter esteem and low end to end postpone when contrasted with other directing conventions in a star topology. Throughput increments exponentially as the movement stack increments. DYMO and DSR have high throughput as they have a high bundle conveyance proportion. AODV expends low vitality in transmit and get mode, however more vitality out of gear mode. Then again ZRP expends more vitality in transmit and get mode, yet low vitality out of gear mode.

Table.2. Comparison of Routing Protocols in Star Topology

\begin{tabular}{|l|c|c|c|c|}
\hline \multicolumn{1}{|c|}{ Parameters } & AODV & DSR & DYMO & ZRP \\
\hline $\begin{array}{l}\text { Packet Delivery Ratio in } \\
\text { percentage }\end{array}$ & 52.67 & 74.38 & 71.399 & 64.81 \\
\hline Average Jitter in seconds & 1.35541 & 2.80947 & 2.24525 & 0.09297 \\
\hline $\begin{array}{l}\text { Average end to end delay in } \\
\text { seconds }\end{array}$ & 4.20775 & 3.839 & 1.93898 & 0.1746 \\
\hline Throughput in Kbps & 115 & 268 & 257 & 235 \\
\hline $\begin{array}{l}\text { Energy consumed in Transmit } \\
\text { mode in mJ }\end{array}$ & 0.14669 & 0.08938 & 0.09188 & 0.13891 \\
\hline $\begin{array}{l}\text { Energy consumed in Receive } \\
\text { mode in mJ }\end{array}$ & 0.09381 & 0.05664 & 0.05732 & .6548 \\
\hline $\begin{array}{l}\text { Energy consumed in Idle } \\
\text { mode in mJ }\end{array}$ & 3.18611 & 3.20609 & 3.2054 & 2.88975 \\
\hline Total Energy consumed in mJ & 3.42661 & 3.35211 & 3.35461 & 4.68346 \\
\hline $\begin{array}{l}\text { Residual Battery capacity in } \\
\text { mAhr }\end{array}$ & 1198.86 & 1198.88 & 1198.88 & 1198.44 \\
\hline
\end{tabular}

In Cluster topology, AODV and DSR have high bundle conveyance proportion and DYMO has a poor Packet conveyance proportion. The correlation of directing conventions in group topology is given in Table 2. The Average Packet conveyance proportion in Cluster topology is around 85 percent. Normal Jitter esteem is high for low activity load and abatements as the movement stack increments. This is a direct result of the expansion in the parcel era rate. ZRP has low normal jitter esteem and low end to end defer when contrasted with other steering conventions in bunch topology. AODV has poor jitter and end to end delay. DYMO has high throughput. DSR and DYMO devour low vitality in transmit and get mode, however out of gear mode ZRP expends low vitality. In general, DSR and DYMO devour low vitality and ZRP expends more vitality. Leftover battery limit is likewise high in DSR and DYMO. In this way in Cluster topology DSR and DYMO perform very much contrasted with other steering conventions.
Table.3. Comparison of Routing Protocols in Cluster Topology

\begin{tabular}{|l|c|c|c|c|}
\hline \multicolumn{1}{|c|}{ Parameters } & AODV & DSR & DYMO & ZRP \\
\hline $\begin{array}{l}\text { Packet Delivery Ratio in } \\
\text { percentage }\end{array}$ & 85.95 & 85.95 & 85.95 & 85.95 \\
\hline $\begin{array}{l}\text { Average Jitter in } \\
\text { seconds }\end{array}$ & 31.0203 & 0.491155 & 0.146139 & 0.019287 \\
\hline $\begin{array}{l}\text { Average end to end } \\
\text { delay in seconds }\end{array}$ & 31.1209 & 0.50046 & 0.159883 & 0.034896 \\
\hline Throughput in Kbps & 187 & 104 & 99 & 100 \\
\hline $\begin{array}{l}\text { Energy consumed in } \\
\text { Transmit mode in mJ }\end{array}$ & 0.222945 & 0.081358 & 0.08021 & 0.138225 \\
\hline $\begin{array}{l}\text { Energy consumed in } \\
\text { Receive mode in mJ }\end{array}$ & 0.26743 & 0.01989 & 0.018097 & 1.04224 \\
\hline $\begin{array}{l}\text { Energy consumed in } \\
\text { Idle mode in mJ }\end{array}$ & 3.13581 & 3.2149 & 3.21551 & 3.00689 \\
\hline $\begin{array}{l}\text { Total Energy } \\
\text { consumed in mJ }\end{array}$ & 3.626185 & 3.31616 & 3.313817 & 4.187355 \\
\hline $\begin{array}{l}\text { Residual Battery } \\
\text { capacity in mAhr }\end{array}$ & 1198.79 & 1198.89 & 1198.9 & 1198.6 \\
\hline
\end{tabular}

\section{Performance ANAlysis OF EDSR}

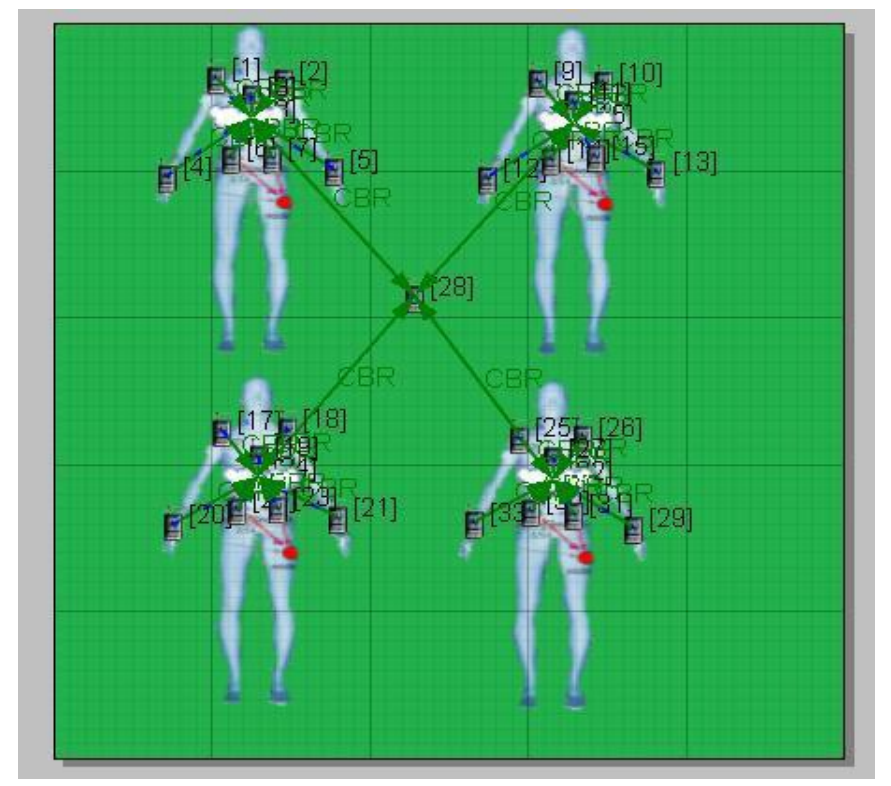

Figure.1. Scenario for WBAN

The execution of EDSR is broke down by fluctuating the activity stack. This is finished by changing the bundle era interim for the movement. For the bundle era interim of 100 milliseconds, ten parcels are sent in one moment and it is said to be substantial activity. Thus for the parcel era interim of 10 seconds just 0.1 bundles are sent in one moment and it is said to be light activity. The situation appeared in Figure 1: is utilized for assessment in view of activity stack and the 
outcomes for parcel conveyance proportion in Figure 2, normal jitter in Figure 3, normal end to end defer in Figure 5, Energy Consumption in Figure 6 are watched. The outcomes obtained for the EDSR is compared with that of the results obtained for DSR.

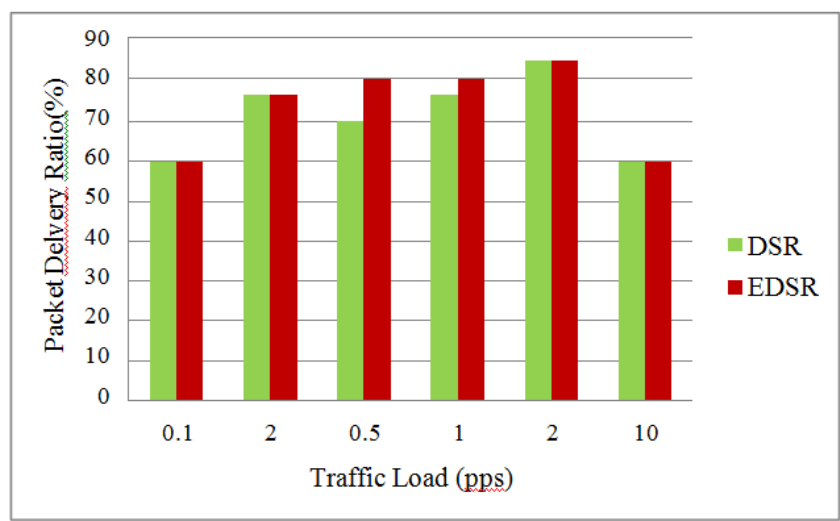

Figure. 2. Packet delivery ratio based on traffic loads

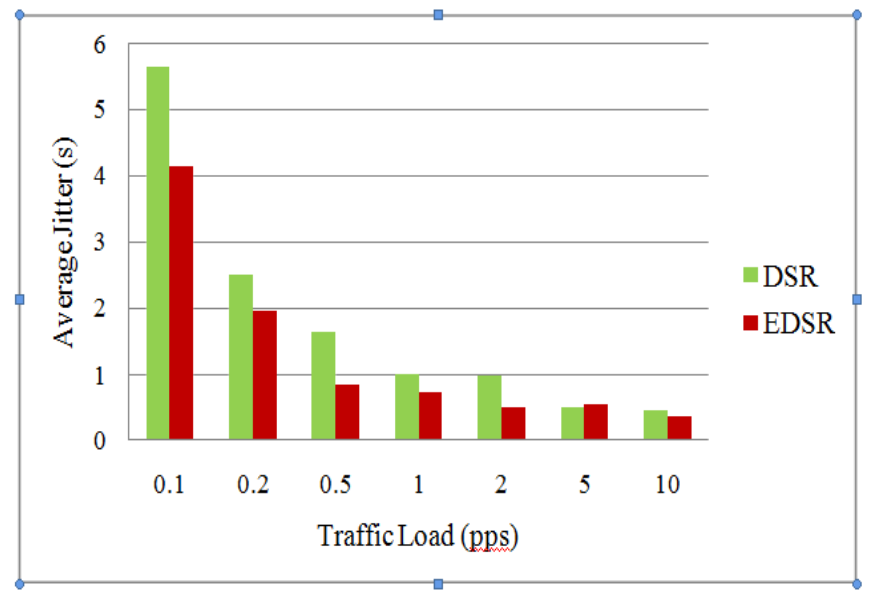

Figure.3. Average jitter based on traffic loads

The parcel conveyance proportion diminishes for the overwhelming activity because of impact of the bundle prompting a parcel drop, while the parcel conveyance proportion diminishes for the light movement because of the expansion in defer prompting parcel drop. At the point when the execution of the EDSR and DSR are viewed as, both the outcomes take after a similar example for different activity stack however the parcel conveyance proportion of EDSR is 12.73 percent higher than that of bundle conveyance proportion of DSR. The normal jitter esteem is low for light movement and high for substantial activity. Be that as it may, when the EDSR and DSR are looked at, the estimation of the normal jitter of the EDSR lessens to about 28.56 percent.
The average end to end delay is low for heavy traffic as increase in the packet generation rate reduces delay. The average end to end delay is high for heavy traffic and this is because when the packet generation rate decreases the delay increases. The average end to end delay increases exponentially as the traffic load increases. When EDSR and DSR are compared the value of the average end to end delay of the EDSR reduces to about 9.64 percent.

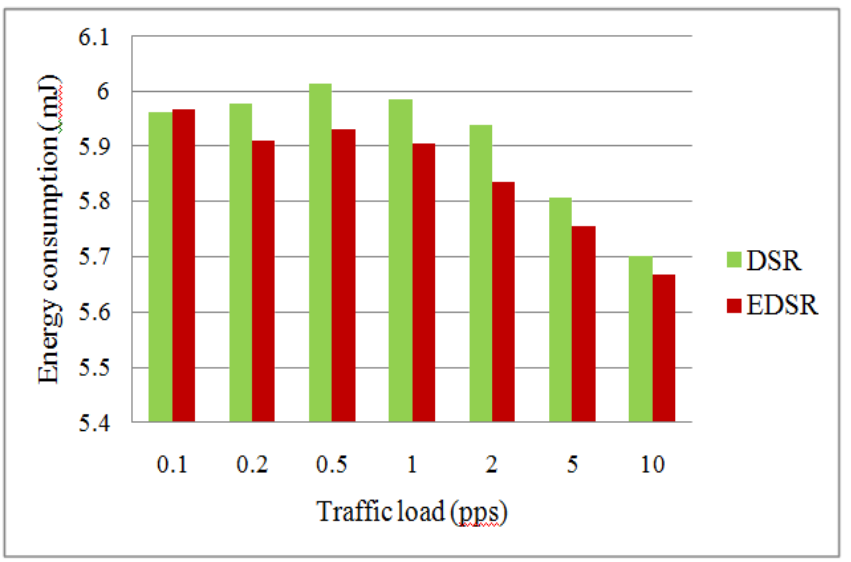

Figure.4. Total Energy Consumption based on Traffic Loads

The vitality utilization is more for light activity and less for overwhelming movement. The vitality utilization is likewise useful for the EDSR when contrasted with the DSR. Vitality expended diminishes exponentially as the movement stack diminishes. Just 89 rate of the vitality required for DSR is adequate for EDSR in the transmit mode and just 87 rate of the vitality required for DSR is adequate for EDSR to work in get mode. All in all, the vitality expended in the EDSR declines to about 11.56 percent when contrasted with DSR.

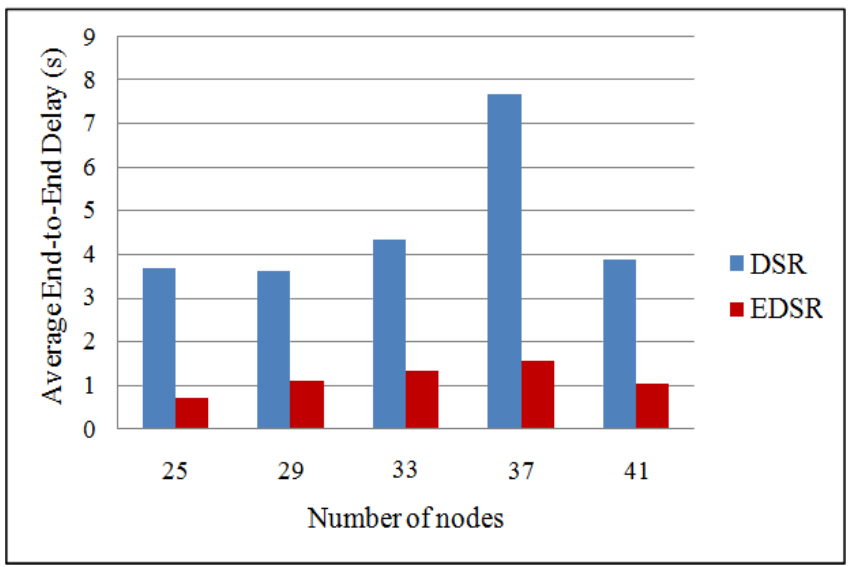

Figure.5.Average End to End Delay Based on Nodes 


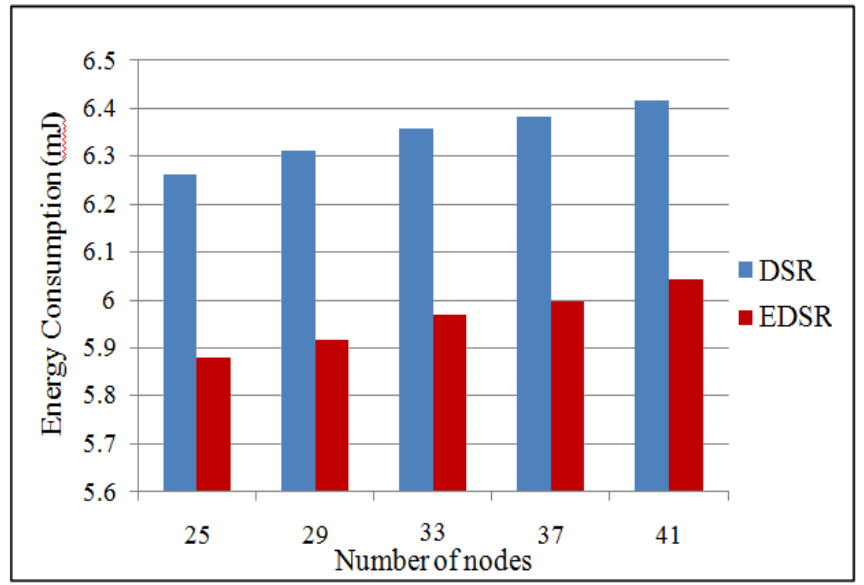

Figure.6. Total Energy Consumption based on Nodes

\section{CONCLUSION}

The performance of EDSR is analyzed by varying the number of nodes in the same scenario. This is done to ensure that the proposed routing protocol EDSR provide better performance even when the network scales in size. By varying the number of nodes in the scenario, the results for average end to end delay and energy consumption are obtained. The delay also decreases to about 28.75 percent in the EDSR when compared to DSR.

The Energy consumed in EDSR is less when compared to the energy consumed in DSR. From the above results, it is clear that the proposed routing protocol perform well even when the numbers of nodes are increased. This increases the residual battery capacity, ensuring the longer life of the batteries.

\section{REFERENCES}

[1] S Gao, Tia. Greenspan, Dan Welsh, Matt Juang, Radford Alm, Alex C.D. "Vital Signs Monitoring and Patient Tracking Over Wireless Network", in Proceedings of the 27th Annual International Conference of the IEEE EMBS 2005.

[2] Bor-Shing Lin; Bor-Shyh Lin; Nai-Kuan Chou; Fok-Ching Chong; Sao-Jie Chen ; C.D. "Real-time Wireless Physiological Monitoring System (RTWPMS)". IEEE Transactions on Information Technology in Biomedicine, Vol. 10, N. 4, 2006.

[3] Milenkovic, Aleksandar ; Otto, Chris ; Jovanov , Emil; C.D. "Wireless Sensor Networks for Personal Health Monitoring: Issues and an Implementation. Computer Communications" 29 2006

[4] Shnayder, Victor; Chen , Bor-rong; Lorincz, Konrad; FulfordJones, Thaddeus R. F; Welsh , Matt C.D. "Sensor Networks For Medical Care". Technical Report TR-08-05, Division of Engineering and Applied Sciences, Harvard University, 2005.

[5] Tia Gao; Tammara Massey; Leo Selavo; David Crawford; Borrong Chen; Konrad Lorincz; Victor Shnayder; Logan Hauenstein; Foad Dabiri; James Jeng; Arjun Chanmugam; David White; Majid Sarrafzadeh; Matt Welsh ; C.D. "The Advanced Health and
Disaster Aid Network: A Light-Weight Wireless Medical System for Triage IEEE Transactions on Biomedical Circuits and Systems, "Vol. 1, No. 32007.

[6] Shanshan Jiang; Yanchuan Cao; Sameer Iyengar; Philip Kuryloski; Roozbeh Jafari Yuan Xue; Ruzena Bajcsy; Stephen Wicker ; C.D. CareNet: "An Integrated Wireless Sensor Networking Environment for Remote Healthcare ".

[7] A. Wood, G. Virone, T. Doan, Q. Cao, L. Selavo, Y. Wu, L. Fang, Z. He, S. Lin, J. Stankovic ; C.D. ALARM-NET: "Wireless Sensor Networks for Assisted-Living and Residential Monitoring", Technical Report CS-2006-13, 2006.

[8] Chin-Feng Lai; Yueh-Min Huang; Jong Hyuk Park; Han-Chieh Chao; C.D. "Adaptative Body Posture Analysis Using Collaborative Multi-Sensors for Elderly Falling Detection." IEEE Intelligent Systems , 2010.

[9] Alexandros Pantelopoulos; Nikolaos G. Bourbakis; C.D. "A Survey on Wearable Sensor-Based Systems for Health Monitoring and Prognosis". IEEE Transactions on Systems, Man, and CyberneticsPart C: Applications and Reviews, Vol. 40, NO. 1, 2010

\section{Authors Profile}

Ms. $R$ Kumari pursed Bachelor of Technology from UPTU of Lucknow, in 2008 and Master of Technology from IET in year 2010. She is Gold Medalist in M.tech. She is currently pursuing Ph.D. and currently working as Assistant Professor in Department of Computer Science, Chitkara University,Chandigarh. She is a member of ACM since 2016. She has published more than 15 research papers in reputed international journals

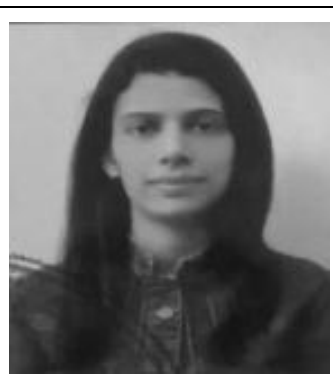
including Thomson Reuters and conferences including IEEE and it's also available online. Her main research work focuses on Software Engineering, Network Security, Data Structure, Big Data Analytics, Data Mining, IoT and Wireless Sensor Network . She has 8 years of teaching experience and 2 years of Research Experience.

Dr Parma Nand is Dean, Computing Science \& Engineering, Galgotias University, Greater Noida. He has over 24 years of teaching and research experience. He has expertise in Wireless and Sensor Network, Cryptography, Algorithm and Computer Graphics. He has been Ex-President of National Engineers Organization.He is senior member of IEEE (USA). He is member

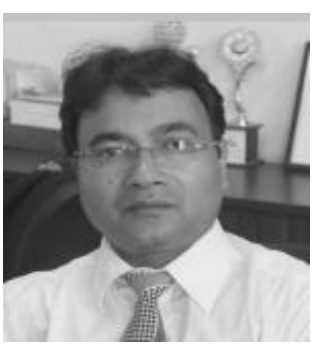
Executive Council of IEEE UP section, member Executive Committee IEEE Computer and Signal Processing Society, member Executive of India council Computer Society, member Executive Council Computer Society of India, Noida section and has acted as an observer in many IEEE conferences. He is also having active memberships of ACM, CSI, ACEEE, ISOC, IAENG, and IASCIT. $\mathrm{He}$ is life time member of Soft Computing Research Society (SCRS) and ISTE. He has published more than 60 papers in peer reviewed international/national journals and conferences. He is an active member of advisory/technical program committee of reputed International/National conferences \& reviewer of number of reputed Journals e.g. Springer, Elsevier Journal Computers \& Electrical Engineering. 